\title{
Membranous Nephropathy and Kimura's Disease Manifesting a Hip Mass. A Case Report with Literature Review
}

\author{
Yoko Obata $^{1-3}$, Akira Furusu ${ }^{1}$, Tomoya Nishino ${ }^{1}$, Hiroshi Ichinose ${ }^{1,3}$, Aki Ohnita ${ }^{4}$, \\ Keisuke Iwasaki ${ }^{5}$, Takashi Taguchi ${ }^{6}$ and Shigeru Kohno ${ }^{1}$
}

\begin{abstract}
We report a case of Kimura's disease with membranous nephropathy. A 15-year-old Japanese boy was referred to our hospital with proteinuria and a subcutaneous mass in the hip. Renal biopsy demonstrated secondary membranous nephropathy (MN) with mild mesangial proliferation and some electron-dense deposits in the mesangium. Laboratory tests revealed eosinophilia and a high level of serum IgE, which are common findings in Kimura's disease. A biopsy of the mass in the hip was performed and Kimura's disease was diagnosed. Treatment with oral prednisolone resulted in a decrease of proteinuria and regression of the subcutaneous mass. Kimura's disease should be included in the differential diagnosis of secondary MN.
\end{abstract}

Key words: membranous nephropathy, Kimura's disease, eosinophilia, hip mass

(Inter Med 49: 1405-1409, 2010)

(DOI: 10.2169/internalmedicine.49.2977)

\section{Introduction}

Kimura's disease is a granulomatous disease of unknown origin that develops in the dermis, subcutaneous tissues, and lymph nodes. It is characterized histologically by the presence of lymphoid follicles, vascular proliferation, and infiltration of eosinophils. The typical presentation is the triad of slowly enlarging, non-tender subcutaneous swelling in the head and neck region, peripheral eosinophilia, and elevated serum IgE levels. Since the report of Kimura et al in 1948 (1), there have been about 120 additional cases described, mostly from Asian countries (2).

The frequency of renal involvement in Kimura's disease, most notably proteinuria and nephrotic syndrome, is apparently high, although the pathogenesis of this association remains unclear (3).

We report an incidental finding of proteinuria that led to the diagnosis of a case of Kimura's disease that also manifested with a subcutaneous mass in the hip. Kimura's dis- ease should be considered in the differential diagnosis of proteinuria with eosinophilia and subcutaneous mass. In addition to discussing this case, we also briefly review the cases of secondary MN involved in Kimura's disease in this manuscript.

\section{Case Report}

A previously healthy 15 -year-old Japanese boy was referred to our hospital in April 2006 due to proteinuria pointed out at the medical check-up in the school. There was no family history of renal disease or congenital abnormalities. On admission, his blood pressure was 111/63 $\mathrm{mmHg}$. He presented no symptoms related to renal diseases (such as edema, fatigue, anorexia, or decreased urine volume) and no clinical findings other than a subcutaneous mass in the left hip. The hip mass was first noted when the patient was 2 years old, and since then had gradually increased in size. The mass was soft, non-tender, and at admission measured about $8 \times 6 \mathrm{~cm}$. No swelling of lymph

\footnotetext{
${ }^{1}$ The Second Department of Internal Medicine, Nagasaki University School of Medicine, Nagasaki, ${ }^{2}$ Career Development Center for Medical Doctor, Nagasaki University Hospital, Nagasaki, ${ }^{3}$ Hokusho Central Hospital, Sasebo, ${ }^{4}$ Ureshino Medical Center, Ureshino, ${ }^{5}$ Sasebo City General Hospital, Sasebo and ${ }^{6}$ Department of Pathology, Nagasaki University Graduate School of Biomedical Sciences, Nagasaki Received for publication October 5, 2009; Accepted for publication March 30, 2010 Correspondence to Dr. Akira Furusu, furusua-ngs@umin.ac.jp
} 

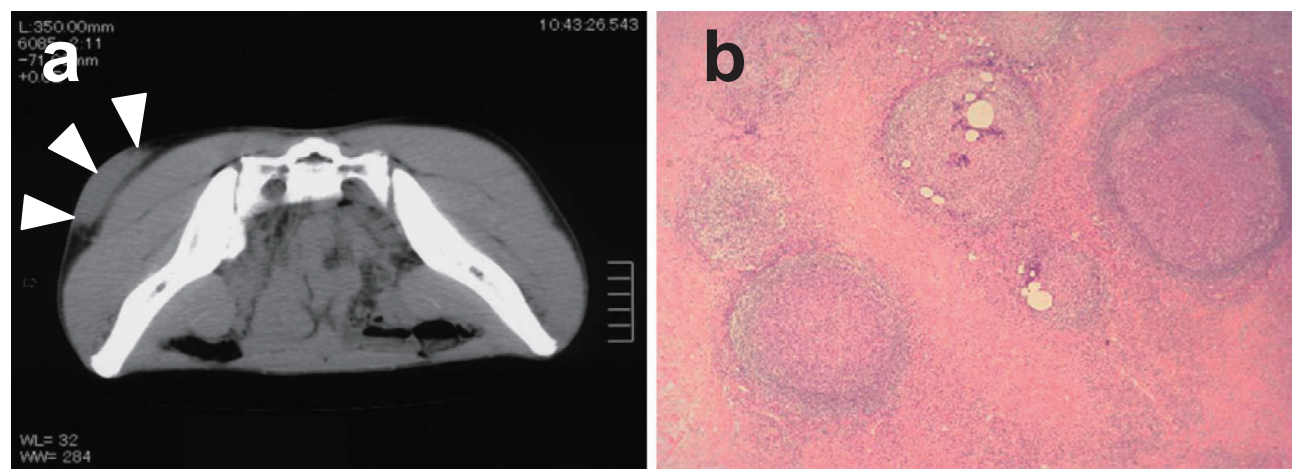

Figure 1. a) Abdominal CT imaging of the subcutaneous mass in the left hip (arrowheads). b) Light microscopy of a sample from skin biopsy (Hematoxylin and Eosin staining, $\times 100)$. Eosinophil infiltration, lymphoid follicles, fibrosis, and vascular proliferation were noted.

nodes was noted. The white blood cell count (WBC) was $8,100 / \mu \mathrm{L}$, with $30.5 \%$ eosinophils. Total protein and albumin were $6.9 \mathrm{~g} / \mathrm{dL}$ and $4.2 \mathrm{~g} / \mathrm{dL}$, respectively. Serum creatinine and blood urea nitrogen (BUN) were in the normal range. Creatinine clearance was $115.7 \mathrm{~mL} / \mathrm{min}$. Urinalysis indicated a urinary protein level of $2.1 \mathrm{~g} /$ day. Urinary occult blood was 3+ in a qualitative test, and 30-50 red blood cells (RBCs) were present per visual field in the sediment. Dysmorphic RBCs were present in the sediment of urine. No urinary cast was noted and urinary $\beta 2$-microglobulin was in the normal range $(103 \mu \mathrm{g} / \mathrm{L})$. Urinary $\mathrm{N}$-acetyl- $\beta$-Dglucosamidase (NAG) was elevated to $14.0 \mathrm{IU} / \mathrm{L}$ among renal tubular disorder markers. In seroimmunological tests, the serum IgE level increased to $8,450 \mathrm{mg} / \mathrm{dL}$. No evidence of collagen disease was found in the laboratory examinations. Computed tomography (CT) showed no marked changes in the kidneys and a localized mass with unclear boundaries in the left hip (Fig. 1a).

Renal biopsy was performed with echo-guidance on the day following admission. Light microscopy showed mild mesangial proliferative glomerulonephritis with thickening of the basement membrane. On periodic acid-methenaminesilver (PAM) staining, a spike lesion was partially noted and vacuolar changes were present at many sites in the glomerular basement membrane (GBM) (Fig. 2a and b). Granular depositions of $\mathrm{IgG}, \mathrm{C} 3$, and $\mathrm{Clq}$ along the GBM were detected using fluorescent antibodies. Electron microscopy revealed electron-dense deposits in the subepithelial area and also in the mesangium (Fig. 2c and d). No eosinophil infiltration was present in the renal biopsy specimens. Secondary MN was diagnosed, in light of the mild mesangial deposits and proliferation.

There was no apparent cause of $\mathrm{MN}$, such as collagen disease, hepatitis virus infection, drugs, or cancer. Because of the subcutaneous mass in the hip, eosinophilia, and the high serum level of IgE, Kimura's disease was suspected as the underlying disease. Biopsy of the subcutaneous mass in the hip was performed, and histological examinations showed eosinophil infiltration, lymphoid follicles, fibrosis, and vascular proliferation (Fig. 1b). The diagnosis of Kimura's disease was made, and the patient was started on an oral prednisolone therapy $(30 \mathrm{mg} /$ day $)$. After 2 months of treatment with prednisolone, proteinuria decreased rapidly to the normal range, hematuria and eosinophilia normalized, and the subcutaneous mass in his hip regressed (Fig. 3). Prednisolone was discontinued 2 years after diagnosis, and at 4 years follow up neither the proteinuria nor the subcutaneous mass in the hip has recurred.

\section{Discussion}

Kimura's disease is a granulomatous disease of dermis, subcutaneous tissues, and lymph nodes. It presents as benign subcutaneous swelling predominantly around the head and neck region. It is characterized histologically by newly developed lymphoid follicles, increased vascularity, and marked infiltration with eosinophils (4). Additional characteristics include marked peripheral eosinophilia and elevated IgE levels (5). This patient presented with marked eosinophilia, elevated IgE levels. The histological pattern of the subcutaneous mass biopsy specimen in this patient was also compatible with Kimura's disease. However, the location of the hip mass was rare.

A high prevalence of nephropathy in Kimura's disease has been reported (16\% of cases), and $78 \%$ of them had nephrotic syndrome (6). Proteinuria has appeared simultaneously with, or later than, the onset of skin lesions in the majority of patients, while several cases have been reported in which proteinuria developed years prior to the appearance of skin lesions (6). The mass in the hip of this patient was first noted when he was 2 years old, and had gradually increased in size since then. Our case did not present nephrotic syndrome at admission, but urinary protein gradually increased. If he had failed to have the medical check-up at school, the nephrotic syndrome might have developed. Although there have been 86 cases of Kimura's disease with renal involvement reported to date, histological findings of the renal biopsies have been described in 54 of the previously reported cases. The most common histological patterns have been $\mathrm{MN}$ (3), mesangial proliferative glomerulonephritis (7), and minimal change disease (8). To our knowledge, 12 of the reported cases had MN, 21 had mesangial prolif- 

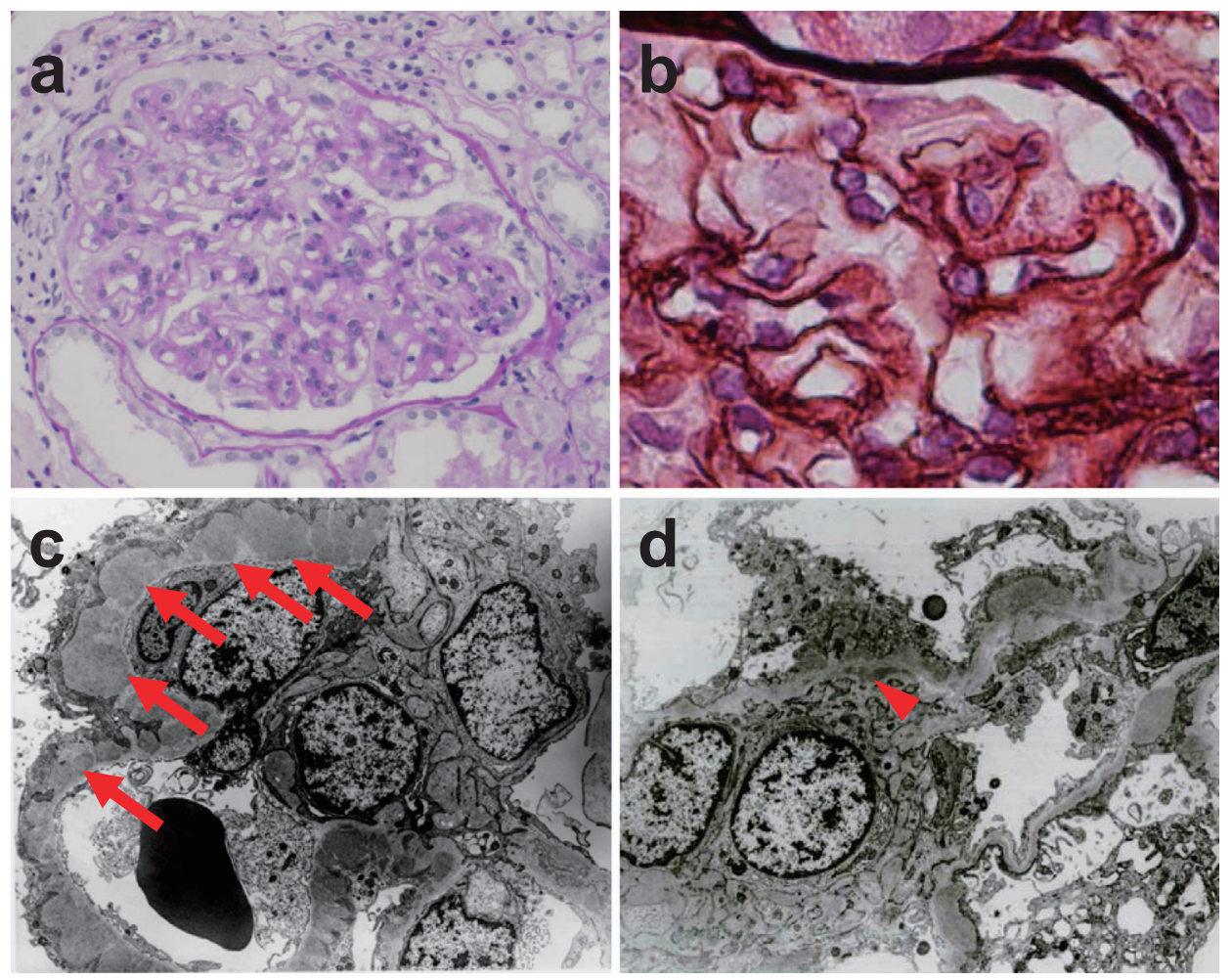

Figure 2. a) Light microscopy of a sample from renal biopsy (Periodic acid-Schiff (PAS) staining, $\times 100)$. The basement membrane was slightly thickened and mild mesangial proliferation was noted. b) Light microscopy of a sample from renal biopsy (Periodic acid-methenamine-silver (PAM) staining, $\times 400)$. Vacular changes and spike lesion were noted. $c$ and d) Electron microscopy of a renal biopsy sample $(\times 3000)$. Deposits were present in the subepithelial regions of glomerular basement membrane (GBM) and mesangial regions. Arrows show subepithelial deposit and the arrowhead shows a mesangial deposit.

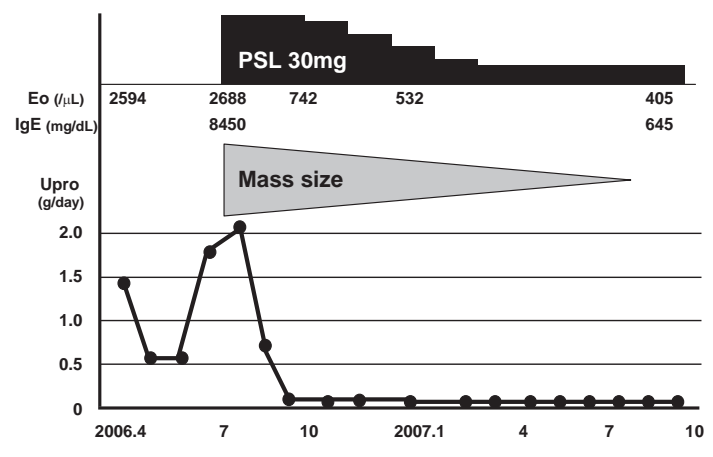

Figure 3. Clinical course. Eo: eosinophils, IgE: immunoglobulin E, PSL: prednisolone, Upro: urinary protein

erative glomerulonephritis, 7 had minimal change disease, and 7 had focal segmental glomerulosclerosis. Our case revealed $\mathrm{MN}$, which is common in histological patterns of renal involvement with Kimura's disease. Table 1 shows the previous reports of secondary $\mathrm{MN}$ due to Kimura's disease $(3,6,9-16)$.

Although the pathogenesis and renal involvement with Kimura's disease is not well understood, several immunopathogenetic features have been noted in this disease. It has been speculated that viral infections or toxins may stimulate the release of lymphokines by altering T-cell immunoregula- tion or by inducing an IgE-mediated type I hypersensitivity. These immunological triggers could result in the characteristic lymph node alterations and the associated renal lesions. Tsukadaira et al described an elevated granulocyte macrophage stimulating factor (GM-CSF), tumor necrosis factor- $\alpha$ $(\mathrm{TNF}-\alpha)$, and soluble interleukin-2 receptor (sIL-2R) in peripheral blood and newly expressed antigens CD4, CD25, and HLA-DR on eosinophils in the patients of Kimura's disease (17). Recently, Katagiri et al measured the expression of cytokines in a patient with Kimura's disease with marked eosinophilia and elevated serum IgE levels. They noted that elevated mRNA levels of IL-5, elevated levels of IL-4 and IL-13, and normal level of interferon-gamma (IFN- $\gamma$ ) (18).

The standard treatment for Kimura's disease is an oral steroid, although termination of the steroid therapy often results in a recurrence of masses (19). Corticosteroids, agents known to modulate lymphokine production and T-cell proliferation, are effective in treating both the proteinuria and Kimura's disease. This effectiveness suggests an immuneregulated disorder where T-cells may play a role in the pathogenesis of both the proteinuria and Kimura's disease. The response to steroids in our patient was effective: the proteinuria decreased and the hip mass was reduced in size.

Several histologic characteristics by electron microscopy (EM) have been identified that may help to distinguish be- 
Table 1. Previous Reports of Secondary Membranous Nephropathy due to Kimura's Disease

\begin{tabular}{|c|c|c|c|c|c|c|c|c|c|c|c|}
\hline Case & Age & Gender & Country & $\begin{array}{l}\text { Upro } \\
\text { (g/day) }\end{array}$ & Hematuria & NS & $\begin{array}{l}\text { Mass } \\
\text { lesion }\end{array}$ & $\begin{array}{c}\text { Eo } \\
(/ \mu \mathrm{L})\end{array}$ & $\begin{array}{c}\lg E \\
(I U / m L)\end{array}$ & $\begin{array}{l}\text { Primary } \\
\text { lesion }\end{array}$ & Treatment \\
\hline Yamada A et al. & 48 & M & Japan & 20 & + & + & retroauricular & 212 & ns & S & PSL,Radiation \\
\hline Kimura O et al. & 57 & M & Japan & 0.89 & - & - & retroauricular & 2088 & $>4000$ & S & PSL \\
\hline Akosa $A B$ et al. & 71 & M & China & 3.8 & + & ns & supraclavicular & 10875 & 720 & $S+R$ & ns \\
\hline Matsuda $O$ et al. & 68 & M & Japan & 4.0 & - & + & $\begin{array}{l}\text { Retroauricular, axillary } \\
\text { Cervical, inguinal }\end{array}$ & 1140 & 12000 & S & PSL \\
\hline Hui PK et al. & 43 & M & China & 22 & ns & + & cervical & 1900 & ns & S & PSL \\
\hline Liu $C$ et al. & 36 & M & China & 14.1 & - & + & submandibular & 616 & 620 & S & PSL, CPA, excision \\
\hline Liu $C$ et al. & 48 & M & China & 5.27 & - & + & submandibular & 1326 & 1378 & S & PSL, excision \\
\hline Wang DY et al. & 42 & M & China & 3.77 & ns & & $\begin{array}{l}\text { Cervical, retroauricular, } \\
\text { Submandibular, forehead }\end{array}$ & 876 & ns & S & PSL,CPA, Radiation \\
\hline Wang DY et al. & 19 & M & China & 14.09 & ns & + & retroauricular & $10.1 \%$ & 620 & S & PSL \\
\hline Danis $R$ et al. & 42 & M & Turkey & 6.0 & ns & + & $\begin{array}{l}\text { Cervical, } \\
\text { retroauricular }\end{array}$ & 2260 & 24800 & S & ns \\
\hline Takebara K et al. & 47 & M & Japan & 17 & - & + & retroauricular & 1710 & 32130 & S & PSL,Radiation \\
\hline Yamamoto $\mathrm{M}$ et al & & M & Japan & $\begin{array}{l}100-250 \\
\mathrm{mg} / \mathrm{dL}\end{array}$ & - & + & Thigh, inguinal & 8160 & ns & $\mathrm{R}$ & PSL,6MP \\
\hline Present case & 15 & M & Japan & 2.1 & + & - & hip & 2430 & 8450 & $\mathrm{~s}$ & PSL \\
\hline
\end{tabular}

Upro: urinary protein, NS: nephrotic syndrome, Eo: eosinophils, IgE: immunogloblin E, M: male, ns: not stated, S: skin lesion, R: renal lesion, PSL: prednisolone, CPA: cyclophosphamide, 6MP: mercaptopurine

tween idiopathic and secondary forms of MN. In idiopathic $\mathrm{MN}$, electron dense deposits on EM are exclusively subepithelial and intramembranous. Secondary forms of $\mathrm{MN}$ are often associated with mesangial and/or subendothelial deposits, which suggest a circulating immune complex $(20,21)$. Therefore, in this patient, Kimura's disease and $\mathrm{MN}$ were thought to be causally related rather than coincidential. To date, glucocorticoid therapy has been known to be effective in patients with clinical and histologic evidence of active inflammation (eg, hematuria and/or proliferative or necrotizing glomerular changes). The findings of light microscopy in the renal biopsy showed mild mesangial proliferative change, so we considered that the treatment of prednisolone was effective for hematuria in this patient.

In summary, we report that the detection of proteinuria led to a diagnosis of a case of Kimura's disease manifesting a subcutaneous mass in the hip. In cases of secondary MN with the presence of subcutaneous masses and eosinophilia, Kimura's disease should be included in the differential diagnosis, and the entire body should be investigated for presence of masses. With the identification of numerous immunopathogenetic features in Kimura's disease, future research should be directed at molecular immunology in order to determine the causative relationship between the pathogenesis and renal involvement with Kimura's disease.

\section{References}

1. Kimura T, Yoshimura S, Ishikawa E. On the unusual granulation combined with hyperplastic changes of lymphatic tissues. Trans Soc Pathol Jpn 37: 179-180, 1948.

2. Danno K, Horio T, Miyachi Y, Hayakawa M, Takatsuki K. Coexistence of Kimura's disease and lichen amyloidosus in three patients. Arch Dermatol 118: 976-980, 1982.

3. Yamada A, Mitsuhashi K, Miyakawa $\mathrm{Y}$, et al. Membranous glomerulonephritis associated with eosinophilic lymphfolliculosis of the skin (Kimura's disease): report of a case and review of the literature. Clin Nephrol 18: 211-215, 1982.
4. Reed RJ, Terazakis N. Subcutaneous angioblastic lymphoid hyperplasia with eosinophilia (Kimura's disease). Cancer 29: 489-497, 1972.

5. Konishi N, Tamura T, Kawai C, Shirai T. IgE associated nephropathy in a patient with subcutaneous eosinophilic lymphoid granuloma (Kimura's disease). Virchows Arch A Pathol Anat Histol 392: 127-134, 1981.

6. Matsuda $\mathrm{O}$, Makiguchi K, Ishibashi K, et al. Long-term effects of steroid treatment on nephrotic syndrome associated with Kimura's disease and a review of the literature. Clin Nephrol 37: 119-123, 
1992.

7. Qunibi WY, Al-Sibai MB, Akhtar M. Mesangio-proliferative glomerulonephritis associated with Kimura's disease. Clin Nephrol 30: 111-114, 1988.

8. Matsumoto K, Katayama H, Hatano M. Minimal-change nephrotic syndrome associated with subcutaneous eosinophilic lymphoid granuloma (Kimura's disease). Nephron 49: 251-254, 1988.

9. Takebara K, Ogawa K, Iijima M, Hino H, Kawashima M, Harada S. Kimura's disease and its renal involvement. Rinsho Derma (Japanese) 22: 1183-1189, 1980.

10. Akosa AB, Sherif A, Maidment CG. Kimura's disease and membranous nephropathy. Nephron 58: 472-474, 1991.

11. Danis R, Ozmen S, Akin D, et al. Thrombosis of temporal artery and renal vein in Kimura-disease-related nephrotic syndrome. J Thromb Thrombolysis 27: 115-118, 2009.

12. Hui PK, Chan YW. Kimura's disease--treatment with steroid. Histopathology 17: 286-287, 1990.

13. Kimura O, Konno T. A case of subcutaneous eosinophilic lymphoid granuloma (Kimura's disease) associated with membranous nephropathy. Nippon Naika Gakkai Zasshi 74: 1759-1762, 1985 (in Japanese).

14. Liu $\mathrm{C}, \mathrm{Hu} \mathrm{W}$, Chen $\mathrm{H}$, et al. Clinical and pathological study of Kimura's disease with renal involvement. J Nephrol 21: 517-525, 2008.
15. Wang DY, Mao JH, Zhang Y, et al. Kimura disease: a case report and review of the Chinese literature. Nephron Clin Pract 111: c55c61, 2009.

16. Yamamoto M, Matsuoka $K$, Kougo $T$, Honda $K$. A case of Kimura's disease with nephrotic syndrome. The Journal of the Japan Pediatric Society 77: 13-20, 1973 (in Japanese).

17. Tsukadaira A, Kitano K, Okubo Y, et al. A case of pathophysiologic study in Kimura's disease: measurement of cytokines and surface analysis of eosinophils. Ann Allergy Asthma Immunol 81: 423-427, 1998.

18. Katagiri K, Itami S, Hatano Y, Yamaguchi T, Takayasu S. In vivo expression of IL-4, IL-5, IL-13 and IFN-gamma mRNAs in peripheral blood mononuclear cells and effect of cyclosporin $\mathrm{A}$ in a patient with Kimura's disease. Br J Dermatol 137: 972-977, 1997.

19. Day TA, Abreo F, Hoajsoe DK, Aarstad RF, Stucker FJ. Treatment of Kimura's disease: a therapeutic enigma. Otolaryngol Head Neck Surg 112: 333-337, 1995.

20. Davenport A, Maciver AG, Hall CL, MacKenzie JC. Do mesangial immune complex deposits affect the renal prognosis in membranous glomerulonephritis? Clin Nephrol 41: 271-276, 1994.

21. Jennette JC, Iskandar SS, Dalldorf FG. Pathologic differentiation between lupus and nonlupus membranous glomerulopathy. Kidney Int 24: 377-385, 1983.

(C) 2010 The Japanese Society of Internal Medicine http://www.naika.or.jp/imindex.html 